\title{
Loperamide: an emerging drug of abuse and cause of prolonged QTC
}

\author{
Authors: George Whittaker ${ }^{A}$ and Joseph Newman ${ }^{B}$
}

Loperamide is an over-the-counter medication which is commonly used to treat diarrhoea. In excessive doses, loperamide acts as an opioid on the central nervous system, which contributes to its increasing popularity as an alternative substance for opiate addictions. High doses for prolonged periods can cause prolonged QTc and provoke life-threatening arrhythmias, such as ventricular fibrillation. We report the case of a young female who developed dangerous arrhythmias as a result of chronic loperamide overdosing. Following syncopal episodes at rest, she was admitted for a period of monitored observation and later discharged with a plan to taper her loperamide in the community. Upon second presentation, her loperamide was replaced with buprenorphine patches, which were then weaned successfully in the community. Despite this, she passed away several months later, most likely from an underlying congenital cardiac arrhythmia which was unmasked by excessive use of loperamide.

KEYWORDS: loperamide, arrhythmias, prolonged QTc, addiction

DOI: $10.7861 /$ clinmed.2020-1046

\section{Case presentation}

A 30-year-old woman presented to the emergency department following two episodes of syncope. She described palpitations and light-headedness while sitting before briefly losing consciousness. The two episodes were witnessed and described as a loss of consciousness that lasted a few seconds and were accompanied by several jerking movements. Recovery was rapid without post-ictal symptoms. She had a psychiatric history including non-epileptic seizures, attention deficit hyperactivity disorder, post-traumatic stress disorder, suicide attempts and a loperamide addiction (100 mg/day). This addiction to loperamide developed secondary to a codeine addiction, with high-dose loperamide used as an opioid substitute. There was no personal history of cardiac disease, however, her paternal grandmother had suffered from Stokes-Adams attacks and died suddenly with no clear

Authors: A specialty trainee year-1 in cardiothoracic surgery, Wythenshawe Hospital, Manchester, UK; ${ }^{B}$ specialty registrar in respiratory medicine, Royal Papworth Hospital, Cambridge, UK explanation. Other than loperamide, her only medication was methylphenidate. Examination was unremarkable. Lying and standing blood pressure measurements were equivalent.

\section{Initial investigations}

Electrocardiography (ECG) showed a dangerously prolonged QTc ranging between 553 ms and 567 ms (Fig 1). First-degree atrioventricular block and right bundle branch block were also present. Previous ECGs from 2015 showed a QTc of 370 ms, which was before the loperamide addiction had started. Routine bloods including electrolytes were all within normal range.

\section{Diagnosis}

A patient and collateral history, physical examination and ECG were performed for initial diagnostic evaluation. True syncope (as opposed to another aetiology) sounded likely due to rapid onset, short duration and spontaneous complete recovery. Psychogenic collapse was considered but unlikely due to the short duration and limited number of episodes. Seizure was also very unlikely due to the lack of preceding aura, post-ictal state or characteristics of myoclonus.

Reflex syncope seemed unlikely as there was no discernible trigger, and orthostatic hypotension was excluded with blood pressure measurements. Cardiac syncope was the most likely cause due to the sudden onset of palpitations immediately preceding the episodes in addition to the prolonged QTc on the initial ECG.

There were no electrolyte abnormalities detected that may have precipitated an arrhythmia. She had no relevant history of cardiac diseases apart from a suspicious family history. She was not on any other QTc-prolonging medications aside from the excessive doses of loperamide, which is known to cause prolonged QTc at high doses. ${ }^{1}$ This process of elimination led to the conclusion that loperamide was the sole precipitant of her syncope.

\section{Initial management and prognosis}

As she was deemed high risk due to preceding palpitations and syncope in the sitting position, she was admitted for 48 -hours observation on a cardiac monitor. She was later reviewed by the cardiology and liaison psychiatry teams who recognised that loperamide was likely to be causing the syncopal episodes, however, she relied on loperamide for avoidance of opiate withdrawal symptoms. It was therefore decided that her 


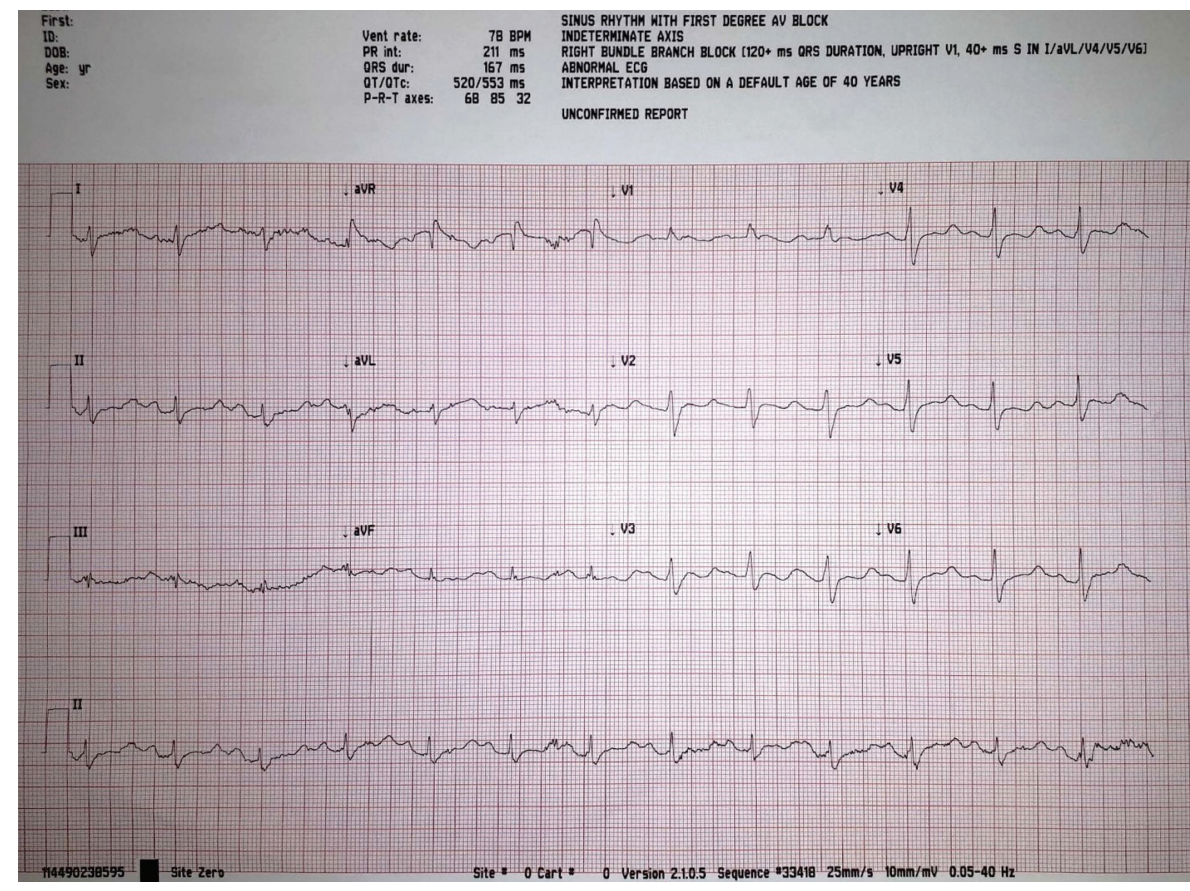

Fig 1. Electrocardiography showing a dangerously prolonged QTc.

loperamide dose should be slowly weaned in the community. She was discharged to the care of the community substance misuse service with advice to consider switching loperamide to buprenorphine. Her prognosis was expected to be favourable with successful detoxification.

\section{Case progression and outcome}

An urgent outpatient echocardiography showed no abnormalities and a Holter monitor showed no significant events. Two weeks after discharge, she re-presented to the emergency department with chest pain, nausea and hyperventilation. As she had tapered to $30 \mathrm{mg} /$ day of loperamide and the ECG showed a borderline QTc of $436 \mathrm{~ms}$, these symptoms were attributed to anxiety triggered by mild opiate withdrawal. The decision was then made to switch the loperamide to buprenorphine patches for weaning in the community.

She was successfully weaned from the buprenorphine patches after 1 year. However, she was still experiencing symptoms on occasion after opiates had been fully withdrawn. An appointment was subsequently made for insertion of an implantable loop recorder. While awaiting the procedure, the patient died.

\section{Discussion}

Extreme doses of loperamide have been reported to provoke dangerous arrhythmias and sudden death. ${ }^{1-3}$ These arrhythmias include prolonged QTc, ventricular fibrillation and torsades des pointes. The daily doses used by the patients in those case reports ranges from $192 \mathrm{mg}$ to $400 \mathrm{mg}$. Interestingly, genetically confirmed Brugada syndrome became apparent on the ECG of an adolescent with chronic loperamide overuse, with subsequent resolution of the Brugada pattern once loperamide was ceased. ${ }^{2}$ This implies that loperamide has the ability to unmask congenital cardiac arrhythmias.
The potential for loperamide to be misused as a substance of abuse is even less recognised, with few reported cases linked to cardiotoxicity. ${ }^{4}$ Evidence for causation is demonstrated in this case by the normal QTc interval prior to the loperamide addiction and the decreased QTc interval following reduction of loperamide intake. Furthermore, due to the family history of Stokes-Adams attacks, this may be a case of subclinical congenital long QT syndrome which was exacerbated by loperamide, as has been described in previous cases. ${ }^{1}$

Patients presenting with syncope should be assessed and treated in accordance with the European Society of Cardiology (ESC) guidelines on syncope. ${ }^{5}$ This should include risk stratification, initial cardiac investigations and appropriate management (including admission for monitored observation in high-risk cases). Additionally, any electrolyte abnormality should be corrected and drugs contributing to prolonged QTc held. Patients with syncope secondary to substance misuse should be managed with joint input from cardiology and liaison psychiatry with experience in substance misuse. Substances of abuse contributing to prolonged QTc should be slowly weaned or substituted in the community with the support of substance misuse services, and outpatient cardiac investigations should be performed with appropriate follow-up.

\section{Summary}

> Loperamide, a common anti-diarrhoeal drug, has the potential for substance misuse and is an emerging public health issue amid the growing opiate crisis.

> High doses of loperamide can act on the central nervous system and cause prolonged QTc, which can precipitate life-threatening arrhythmias and sudden death.

> Loperamide overdose should be considered in patients with a history of opiate addiction who present with syncope and preceding palpitations. 
$>$ Patients presenting with cardiac syncope secondary to loperamide overdose should be admitted for observation and urgent electrophysiological investigations, with joint input from cardiology and psychiatry.

> Other drugs known to prolong QTc should be held where possible and electrolyte abnormalities checked for and corrected.

\section{References}

1 Upadhyay A, Bodar V, Malekzadegan M et al. Loperamide induced life threatening ventricular arrhythmia. Case Rep Cardiol 2016;2016:5040176

2 Stefek B, Wolfe LT, Cohen M. Brugada syndrome associated with adolescent loperamide abuse. Pediatrics 2018;142:e20181423.
3 Eggleston W, Clark KH, Marraffa JM. Loperamide abuse associated with cardiac dysrhythmia and death. Ann Emerg Med 2017: 69:83-6.

4 Smith NA, Sehring M, Chambers J. Loperamide abuse and cardiotoxicity. J Community Hosp Intern Med Perspect 2017;7:275.

5 Brignole M, Moya A, de Lange F] et al. 2018 ESC Guidelines for the diagnosis and management of syncope. Eur Heart J 2018:39:1883-948.

Address for correspondence: Dr George Whittaker, Department of Cardiothoracic Surgery, Wythenshawe Hospital, Manchester University NHS Foundation Trust, Manchester M23 9LT, UK. Email: george.whittaker@nhs.net 